\title{
Linearized Gyro-Kinetic Equation
}

\author{
Peter J. Catto \\ Kang T. Tsang
}

\section{MASTER}

\section{OAK RIDGE NATIONAL LABORATORY}




\section{DISCLAIMER}

This report was prepared as an account of work sponsored by an agency of the United States Government. Neither the United States Government nor any agency Thereof, nor any of their employees, makes any warranty, express or implied, or assumes any legal liability or responsibility for the accuracy, completeness, or usefulness of any information, apparatus, product, or process disclosed, or represents that its use would not infringe privately owned rights. Reference herein to any specific commercial product, process, or service by trade name, trademark, manufacturer, or otherwise does not necessarily constitute or imply its endorsement, recommendation, or favoring by the United States Government or any agency thereof. The views and opinions of authors expressed herein do not necessarily state or reflect those of the United States Government or any agency thereof. 


\section{DISCLAIMER}

Portions of this document may be illegible in electronic image products. Images are produced from the best available original document. 
Printed in the United States of America. Available from National Technical Information Service

U.S. Department of Commerce

5285 Port Royal Road, Springfield, Virginia 22161

Price: Printed Copy $\$ 5.00$; Microfiche $\$ 2.25$

This report was prepared as an account of work sponsored by the United States Government. Neither the United States nor the Energy Research and Development Administration, nor any of their employees, nor any of their contractors, subcontractors, or their empioyees, makes any warranty, express or implied, or assumes any legal liability or responsibility for the accuracy, completeness or usefulness of any information, apparatus, product or process disclosed, or represents that its use would not infringe privately owned rights. 
ORNL/TM-5237

Contract No. W-7405-eng-26

Thermonuclear Division

\section{LINEARIZED GYRO-KINETIC EQUATION}

Peter J. Catto

Department of Mechanical and Aerospace Sciences

University of Rochester, Rochester, N.Y. 14627

and

Kang T. Tsang

Oak Ridge National Laboratory, Oak Ridge, Tennessee 37830

(To be published in Physics of Fluids)

JANUARY 1976

NOTICE This document contains information of a preliminary nature and was prepared primarily for internal use at the Oak Ridge National Laboratory. It is subject to revision or correction and therefore does not represent a final report.

OAK RIDGE NATIONAL LABORATORY

Oak Ridge, Tennessee 37830 operated by

UNION CARBIDE CORPORATION

for the

U.S. ENERGY RESEARCH AND DEVELOPMENT ADMINISTRATION

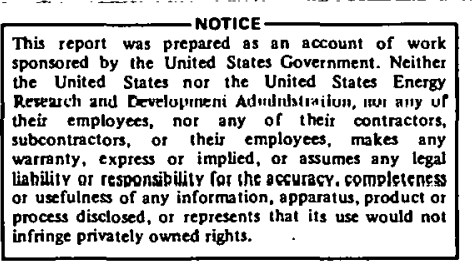




\title{
LINEARIZED GYRO-KINETIC EQUATION *
}

\author{
Peter J. Catto \\ Department of Mechanical and Aerospace Sciences \\ University of Rochester, Rochester, N.Y. 14627. \\ Kang T. Tsang \\ Oak Ridge National Laboratory \\ Oak Ridge, Tënnessee 37830
}

\begin{abstract}
An ordering of the linearized Fokker-Planck equation is performed in which gyroradius corrections are retained to lowest order and the radial dependence appropriate for sheared magnetic fields is treated without resorting to a WKB technique. This description is shown to. be necessary to obtain the proper radial dependence. when the product of the poloidal wavenumber and the gyroradius is large ( $k \rho \gg 1)$. A like particle collision operator valid for arbitrary $k \rho$ also has been derived. In addition, neoclassical, drift, finite $\beta$ (plasma pressure/magnetic pressure), and unperturbed toroidal electric field modifications are treated.
\end{abstract}

\footnotetext{
* This work was supported by the U. S. Energy Research and Development Administration by contract $E(11-1)-3497$ at Rochester ( $P$. J. Catto) and under contract with Union Carbide Corporation at the Oak Ridge National Laboratory (K. T. Tsang). P. J. Catto performed part of his research at Dak Ridge.
} 
INTRODUCTION

For a complete evaluation of the growth rates of drift instabilities in sheared magnetic fields, finite gyroradius effects must be carefully retained. In slab geometry finite gyroradius corrections can result in destabilizing contributions to the growth rate. ${ }^{1-2}$ The addition of finite B (plasma pressure/magnetic pressure) results in considerable complication even in slab geometry, and for a sheared magnetic field has only been evaluated when the gyroradius correction is small. 3-4 In addition, the extension of finite gyroradius effects to sheared toroidal geometry (Tokamaks) may permit the coupling of a mode localized about a rational surface to modes localized about adjacent rational surfaces. The coupling is expected to occur because the group velocity associated with one of these localized modes is away from the rational surface and, at marginal stability, the mode structure extends to distances where ion Landau damping occurs. ${ }^{1-4}$ Present calculations which include finite gyroradius effects assume isolated rational surfaces. ${ }^{4-6}$

In order to treat arbitrary values of the product of poloidal wavenumber $k$ and the ion gyroradius $\rho_{j}$ in sheared magnetic fields, a generalization of the technique of Rutherford and Frieman ${ }^{7-8}$ is employed. The technique retains gyroradius corrections in the lowest order equations for the linearized distribution function. This same gyro-kinetic ordering is employed herein, however, rather than treat the radial and poloidal wave variation via a WKB or eikonal method, an explicit toroidal model with concentric magnetic surfaces is treated. This model permits the toroidal variation to be Fourier decomposed and the derivatives with respect to radial variation to be retained explicitly. 
As a result, magnetic shear effects can be properly treated. The result for large $k \rho_{j}$ is in agreement with previous slab results, ${ }^{10}$ but differs from the WKB treatment which is shown to provide only the local result. The gyro-kinetic formalism also permits neoclassical, finite $\beta$, and unperturbed toroidal electric field $\underline{E}_{0}$ contributions to be retained. The neoclassical corrections are shown to result in negligible modifications. The gyro-kinetic equation valid for $\underline{E}_{0} \neq 0$ and finite $B$ is derived.

For completeness, the $\mathrm{k}_{j}$ modification of the ion-ion Fokker-Planck collision operator is considered. The result is quite complex for arbitrary $k \rho_{j}$; however, for $k \rho_{i} \gg 1$ it can be reduced to a particularly simple form. This gyro-kinetic ordering could also be employed to determine the gyroradius modifications of MHD instabilities. 


\section{GYRO-KINETIC ORDERING AND EQUATIONS}

Working in a toroidal geometry with circular, concentric flux surfaces, the unperturbed magnetic field may be written as $\underline{B}_{0}=\left(B_{0} R_{0} / R\right)\{\underline{\hat{\zeta}}+[\varepsilon / q(r)] \underline{\hat{\theta}}\}$; with $\varepsilon=r / R_{0}, R=R_{0}(1+\varepsilon \cos \theta), \theta$ and $\zeta$ the poloidal and toroidal angle variables, $q$ the safety factor, and $r$ the radial variable measured from the center of the concentric flux circles located at a distance $R_{0}$ from the axis of symmetry.

Employing the velocity variables $\mu, E$, and $\phi$ defined by

$$
\begin{aligned}
& \mu=v_{\perp}^{2} / 2 B \quad E=\frac{1}{2}\left(v_{11}^{2}+v_{1}^{2}\right)=\frac{1}{2} v^{2} \\
& \underline{v}=v_{11} \underline{\hat{n}}+v_{\perp}(\underline{\hat{r}} \cos \phi+\underline{\hat{e}} \sin \phi)=v_{11} \underline{\hat{n}}+\underline{v}_{\perp}=v_{11} \underline{\hat{n}}+v_{\perp} \underline{\hat{v}} \underline{\underline{ }} \\
& \underline{\hat{e}}=\underline{\hat{n}} \times \underline{\hat{r}} \quad \underline{\hat{\Phi}}=\underline{\hat{n}} \times \underline{\hat{v}} \underline{I} \\
& \nabla_{v}=\underline{v} \frac{\partial}{\partial E}+\underline{v}_{\perp} \frac{1}{B} \frac{\partial}{\partial \mu}+\hat{\phi} \frac{1}{v_{\perp}} \frac{\partial}{\partial \phi} \\
& B=\left|\underline{B}_{0}\right| \quad \underline{\hat{n}}=\underline{B}_{0} / B \quad \Omega=Z \mathrm{eBB} / \mathrm{MC},
\end{aligned}
$$

denoting the unperturbed and perturbed distribution functions by $F$ and $f$, and writing $F=F_{M}+F_{D}$ as the sum of Maxwellian $F_{M}$ and diamagnetic $F_{D}$ contributions,

$$
\begin{aligned}
& F_{M}=F_{M}(r, E)=N(r)\left[\frac{M}{2 \pi T(r)}\right]^{\frac{3}{2}} \exp [-M E / T(r)] \\
& F_{D}=F_{D}(r, \theta, \mu, E, \phi)=\frac{1}{\Omega} \underline{v} \times \underline{\hat{n}} \cdot \nabla F_{M},
\end{aligned}
$$

the linearized kinetic or Fokker-Planck equation may be written conveniently by including gyrophase independent portion of $F, F_{M}$, inside $\underline{v} \cdot \nabla$ to obtain 


$$
\begin{gathered}
\frac{\partial f}{\partial t}+\left[\underline{v} \cdot \nabla-\Omega \frac{\partial}{\partial \phi}\right]\left[f+\frac{Z e F_{M}}{T} \Phi\right]-Z e v_{\perp} \Phi \cos \phi \frac{\partial}{\partial r}\left(\frac{F_{M}}{T}\right) \\
-\frac{Z e}{M} \nabla \Phi \cdot \nabla_{v} F_{D}+\dot{\mu} \frac{\partial f}{\partial \mu}+\dot{\phi} \frac{\partial f}{\partial \phi}=C\{f\},
\end{gathered}
$$

where

$$
\begin{aligned}
& B \dot{\mu}=-\underline{v} \cdot \nabla B+v_{\perp} v_{11}\left\{v_{11}\left[\underline{\hat{n}} \cdot \nabla \underline{\hat{n}} \cdot \underline{\hat{v}}_{\perp}\right]-v_{\perp}\left[\underline{\hat{r}} \cdot \nabla \underline{\hat{r}} \cdot \underline{\hat{n}} \cos ^{2} \phi\right.\right. \\
& \left.\left.+\underline{\hat{e}} \cdot \nabla \underline{\hat{e}} \cdot \underline{\hat{n}} \sin ^{2} \phi+(\underline{\hat{e}} \cdot \nabla \underline{\hat{r}} \cdot \underline{\hat{n}}+\underline{\hat{r}} \cdot \nabla \underline{\hat{e}} \cdot \underline{\hat{n}}) \sin \phi \cos \phi\right]\right\} \\
& \dot{\phi}=v_{11} \underline{\hat{n}} \cdot \nabla \underline{\hat{e}} \cdot \underline{\hat{r}}-\left(v_{11}^{2} / v_{\perp}\right) \underline{\hat{n}} \cdot \nabla \underline{\hat{n}} \cdot \underline{\hat{\phi}}+v_{11}\left[\underline{\hat{r}} \cdot \nabla \underline{\hat{e}} \cdot \underline{\hat{n}} \cos ^{2} \phi\right. \\
& \left.=\underline{\hat{e}} \cdot \nabla \underline{\hat{r}} \cdot \underline{\hat{n}} \sin ^{2} \phi+(\underline{\hat{e}} \cdot \nabla \underline{\hat{e}} \cdot \underline{\hat{n}}-\underline{\hat{r}} \cdot \nabla \underline{\hat{r}} \cdot \underline{\hat{n}}) \sin \phi \cos \phi\right] \\
& -v_{\perp}[\underline{\hat{r}} \cdot \nabla \underline{\hat{r}} \cdot \underline{\hat{e}} \cos \phi .-\underline{\hat{e}} \cdot \nabla \underline{\hat{e}} \cdot \underline{\hat{r}} \sin \phi] .
\end{aligned}
$$

In eq. (1), $\Phi$ is the perturbed electrostatic potential, $N$ and $T$ are the unperturbed number density and temperature of the species, and $C[f\}$ represents the linearized Fokker-Planck collision operator; the quantities e and $c$ are the magnitude of the charge on an electron and speed of light, while $M$ and $Z$ are the species mass and charge in units of $e^{-} Z=-1$ for electrons).

Taking $f$ and $\Phi$ to be of the form

$$
\left[\begin{array}{l}
f \\
\Phi
\end{array}\right]=\left[\begin{array}{c}
\hat{f}(r, \theta, \underline{v}) \\
\hat{\Phi}(r, \theta)
\end{array}\right] \exp (-i \omega t+i m \theta-i \ell \zeta)
$$

where the fast $\theta$ dependence is explicitly indicated $(m>>1)$ and the: hatted quantities contain only slow $\theta$ dependence, then neglecting the distinction between $R$ and $R_{0}$ except under derivatives results in

$$
\begin{aligned}
\nabla[\hat{Q}(r, \theta) \exp (i m \theta-i \ell \zeta)] & \approx \exp (i m \theta-i \ell \zeta)\left[\underline{\hat{r}} \frac{\partial}{\partial r}+\hat{\theta}\left(\frac{i m}{r}+\frac{1}{r} \frac{\partial}{\partial \theta}\right)-\underline{\hat{\zeta}} \frac{i \ell}{R_{0}}\right] \hat{Q} \\
& \approx \exp (i m \theta-i \ell \zeta)\left[\nabla_{f}+\nabla_{s}\right] \hat{Q},
\end{aligned}
$$


with

$$
\begin{aligned}
& \nabla_{f}=\underline{\hat{r}} \frac{\partial}{\partial r}+i \underline{\underline{e}} \underline{\hat{n}} \cdot \underline{\hat{\zeta}}\left(\frac{m}{r}+\frac{l \varepsilon}{q R_{0}}\right) \approx \underline{\hat{r}} \frac{\partial}{\partial r}+\underline{\hat{e}} i k \\
& \nabla_{s}=\hat{\theta} \frac{1}{r} \frac{\partial}{\partial r}+i \underline{\hat{n}} \hat{n} \cdot \underline{\hat{\zeta}}\left(\frac{m-l q}{q R_{0}}\right) \approx \underline{\hat{\theta}} \frac{1}{r} \frac{\partial}{\partial \theta}+\underline{\hat{n}} i k_{11}
\end{aligned}
$$

and

$$
k=m / r \quad k_{11}=(m-\ell q) / q R_{0} \cdots
$$

If the distinction between $R$ and $R_{0}$ is retained, then in order to describe, perturbed electric fields that vanish at a rational surface $\left(\oint_{c} d \underline{-}-\underline{E}=0\right.$ along a closed field) a sum over poloidal wavenumbers is required. This complication is a limitation of the concentric magnetic surface model, but could be removed by employing a more sophisticated coordinate system. For simplicity and ease of presentation the concentric magnetic surface model is employed with order $\varepsilon$ corrections neglected. Order $\varepsilon^{1 / 2}$ corrections, which are associated with the distinction between trapped and circulating particles, are retained.

With the notation of eqs. (2) and (3), a gyro-kinetic ordering of eq. may be performed conveniently. This ordering proceeds by assuming that in eq. (1) the $\left[\underline{v} \cdot \nabla_{f}-\Omega \partial / \partial \phi\right]\left[\hat{f}+\left(Z e F_{M} \hat{\Phi} / T\right)\right]$ terms dominate, rather than just the $\Omega \partial / \partial \phi\left[\hat{f}+\left(\operatorname{ZeF}_{M} \hat{\Phi} / T\right)\right]$ term of the more familiar drift-kinetic ordering. Formally, the gyro-kinetic ordering corresponds to an expansion in the small parameters $|\omega /(\Omega \partial \ln \hat{Q} / \partial \phi)|$ and $\left|\underline{v} \cdot \nabla_{s} \hat{Q} / \underline{v} \cdot \nabla_{f} \hat{Q}\right|$.

In order to obtain a particularly simple representation it is desirable to retain certain small terms to lowest order. Expanding $\hat{f}$ and $\hat{\Phi}$ in powers of the small parameters, $\hat{f} \rightarrow \hat{f}+\delta \hat{f}+\ldots$ and $\hat{\Phi} \rightarrow \hat{\Phi}+\delta \hat{\Phi}+\ldots$; and retaining these small terms, the lowest order equation is taken to be 


$$
\left\{\left[\tilde{v} \underline{\hat{r}}_{\perp} \cos \phi+v_{\perp} \underline{\hat{e}} \sin \phi\right] \cdot \nabla_{f}-\Omega \frac{\partial}{\partial \phi}+i \tilde{v}_{\perp} \cos \phi \frac{\partial L}{\partial r}\right\}\left[\hat{f}+\frac{Z_{e} F_{M}}{T} \hat{\Phi}\right]=0,
$$

where $L \equiv\left(k v_{1} / \Omega\right) \cos \phi$ and $\tilde{v}_{\perp}=v_{1} /\left[1+\sin \phi \partial\left(v_{1} / \Omega\right) / \partial r\right]$. The particular virtue of this lowest order form is that the change of variables ${ }^{9}$ $r^{\prime}=r+\left(v_{\alpha} / \Omega\right) \sin \phi, \theta^{\prime}=\theta, \phi^{\prime}=\phi, \mu^{\prime}=\mu$, and $E^{\prime}=E$ applied to eq. (4) results in

$$
\frac{\partial}{\partial \phi^{T}}\left\{\left[\hat{f}+\frac{Z \mathrm{eF}_{M}}{T} \hat{\Phi}\right] \exp (i L)\right\}=0
$$

Consequentiy,

$$
\begin{aligned}
\hat{f}+\frac{Z e F_{M}}{T} \hat{\Phi} & =\hat{g}^{\prime} \exp (-i L) \\
& =\hat{g}\left(r+\frac{v_{1}}{\Omega} \sin \phi, \theta, \mu, E\right) \exp (-i L),
\end{aligned}
$$

that is, $\hat{g}^{\prime}$ is independent of $\phi^{\prime}$.

In obtaining the next higher order equation from eq. (1), small terms are again added in so that the transformation to the primed variables results in a tota' $\phi^{\prime}$ derivative of $\delta \hat{f}+\left(\operatorname{ZeF}_{M} \hat{\delta} \hat{\Phi} / T\right)$. In addition, the small terms added in to lowest order must be corrected for by subtracting them out in this order. The resulting equation is then multiplied by $\Omega^{-7} \exp (i L)$ and a gyrophase averagc over the primed gyrophase $\phi^{\prime}$ performed,

$$
<\cdot>=\frac{1}{2 \pi} \cdot \int_{0}^{2 \pi} \mathrm{d} \phi^{\prime}(\cdots) \text {. }
$$

The result is

$$
\begin{gathered}
\left\langle\frac { 1 } { \Omega } \left\{-i \omega+\left[\underline{v} \cdot \nabla_{s}-i \frac{v_{\perp}}{\Omega} \sin \phi \frac{\partial L}{\partial \theta}\right]+\dot{\mu}\left[\frac{\partial}{\partial \mu}-i \frac{\partial L}{\partial \mu}\right]+\dot{\phi}\left[\frac{\partial}{\partial \phi}-i \frac{\partial L}{\partial \phi}\right]\right.\right. \\
\left.\left.-\left[i \tilde{v}_{\perp} \cos \phi \frac{\partial L}{\partial r}+\left(\tilde{v}_{\perp}-v_{\perp}\right) \cos \phi\left(\frac{\partial}{\partial r}-i \frac{\partial L}{\partial r}\right)\right]\right\}\right\rangle \hat{g}^{\prime} \\
-\left\langle\frac{1}{\Omega} \exp (i L) C\left\{\hat{g}^{\prime} \exp (-i L)\right\}\right\rangle=-\left\langle\frac{i \operatorname{ef} F_{M}}{\Omega T}\left(\omega-\omega_{\star}^{\top}\right) \hat{\Phi} \exp (i L)\right\rangle,
\end{gathered}
$$


where

$$
\begin{aligned}
\omega_{\star}^{\top} \equiv \frac{k T}{M \Omega F_{M}} \frac{\partial F_{M}}{\partial r} & =\frac{k T}{M \Omega N} \frac{\partial N}{\partial r}\left[1+\eta\left(\frac{M E}{T}-\frac{3}{2}\right)\right] \\
\eta & =d \ln T / d \ln N ;
\end{aligned}
$$

$\nabla_{f} \hat{\Phi} \cdot \nabla_{v} F_{D}$ has been rewritten by employing

$$
\nabla_{v} F_{D}=\Omega^{-T}\left[\underline{e} \frac{\partial F_{M}}{\partial r}-M v_{\perp} \underline{\sin \phi} \frac{\partial}{\partial r}\left(\frac{F_{M}}{T}\right)\right] \text {, }
$$

and

$$
\left[\hat{\phi} \cos \phi-\Omega^{-1} \sin \phi \underline{v} \cdot \nabla_{f} \hat{\phi}\right] \approx \frac{\partial}{\partial \phi^{\prime}}[\hat{\phi} \sin \phi \exp (i L)]
$$

has been employed, so that contributions from $\partial\left(F_{M} / T\right) / \partial r$ terms may be safely neglected.

To evaluate the $\phi^{\prime}$ integrals in eq. (6) the integrands must be expressed in terms of the primed variables. Oniy the $r$ dependence presents a problem; however, the usual assumption ${ }^{1-6}$. that the gyroradius is small compared to scale lengths and radial wavelengths permits any function of $r, Q(r)$, to be expanded about $r^{\prime}$ with the result that $Q(r)=Q\left(r^{\prime}\right)+\left(r^{\prime}-r\right) \partial Q / \partial r^{\prime} \cdots$.

Evaluating the integrals in the first angular brackets on the left hand side of eq. (6) by neglecting corrections of order $v_{1} / \Omega r$ compared to one results in the familiar curvature and gradient $B$ drifts plus the parallel velocity correction. The curvature drift is recovered precisely (because it contains no slow $\theta$ dependence), while the gradient B drift and parallel velocity correction are reproduced to within terms of order $\mathrm{m}^{-1}$. Again by neglecting corrections of order $v_{\alpha} / \Omega r$ the right hand side of eq. (6) can be evaluated by retaining $\partial^{2} \hat{\Phi} / \partial r^{\prime 2}$ terms from the gyroradius expansion of $\left(\omega-\omega_{\star}^{\top}\right) \hat{\Phi}$ and employing $\langle\exp (i L)\rangle=J_{0}\left(k v_{\perp} / \Omega\right)$ and $\left\langle\sin ^{2} \phi \exp (i L)\right\rangle=$ $\left(\Omega / k v_{2}\right) J_{1}\left(k v_{1} / \Omega\right)$ 
As a result of the preceding, eq. (6) yields

$$
\begin{aligned}
& \left\{-i \omega+\left[v_{11}+\frac{\mu B}{\Omega} \underline{\hat{n}} \cdot \nabla \times \underline{\hat{n}}\right] \underline{\hat{n}} \cdot \nabla_{s}+\underline{\hat{n}} \times\left[\frac{v_{11}^{2}}{\Omega} \underline{\hat{n}} \cdot \nabla \underline{\hat{n}}+\frac{\mu}{\Omega} \nabla B\right] \cdot \nabla_{f}\right\} \hat{g}^{\prime} \\
& -\left\langle\exp (i L) C\left\{\hat{g}^{\prime} \exp (-i L)\right\}\right\rangle=-\frac{i Z e F_{M}}{T}\left(\omega-\omega_{*}^{\top}\right)\langle\hat{\Phi} \exp (i L)\rangle,
\end{aligned}
$$

where $\underline{\hat{n}} \cdot \nabla \times \underline{\hat{n}}=[\partial(\varepsilon / q) / \partial r]+\left(1 / q_{0}\right)$ and

$$
\begin{aligned}
\langle\hat{\Phi}(r) \exp (i L)\rangle= & J_{0}\left(k v_{\perp} / \Omega\right) \hat{\Phi}\left(r^{\prime}\right)+J_{1}\left(k v_{\perp} / \Omega\right) \frac{v_{\perp}}{2 k \Omega} \frac{\partial^{2} \hat{\phi}}{\partial r^{\prime}{ }^{2}} \\
= & J_{0}\left(k v_{\perp} / \Omega\right)\left[\hat{\Phi}(r)+\frac{v_{\perp}}{\Omega} \sin \phi \frac{\partial \hat{\Phi}}{\partial r}+\frac{v_{1}{ }^{2}}{2 \Omega^{2}} \sin ^{2} \phi \frac{\partial^{2} \hat{\Phi}}{\partial r^{2}}\right] \\
& +J_{1}\left(k v_{\perp} / \Omega\right) \frac{v_{1}}{2 k \Omega} \frac{\partial^{2} \hat{\phi}}{\partial r^{2}} .
\end{aligned}
$$

The latter form of eq. (8) follows upon changing from the prime variables back to the unprimed variables. This final form for $\langle\hat{\Phi} \exp (i L)\rangle$ agrees with the result obtained from slab models in sheared magnetic fields ${ }^{10}$ but differs from the result obtained from a WKB or eikonal treatment of the radial variation of $\hat{\Phi} .^{8}$ Such WKB treatments give $\langle\hat{\Phi} \exp (i L)\rangle=J_{0}\left(k_{\perp} v_{\perp} / \Omega\right) \hat{\Phi}$ where $k_{\perp}^{2}$ is often interpreted as $k^{2}-\partial^{2} / \partial r^{2}$. Consequently, the proper result is obtained from a WKB treatment only in the local (shearless) limit. Note also that the factor $\exp (-i L)$ in eq. (5) results in additional Bessel functions when moments of $\hat{f}$ are formed. In particular,

$$
\int d^{3} v F_{M}\langle\hat{\Phi} \exp (i L)\rangle \exp (-i L)=N \exp (-b) I_{0}(b)\left\{\hat{\Phi}+\left[1-\frac{\partial}{\partial b} \ln I_{0}(b)\right] \frac{v_{I}^{2}}{\Omega^{2}} \frac{\partial^{2} \hat{\phi}}{\partial r^{2}}\right\} \text {, }
$$

where $b \equiv k^{2} v_{T}^{2} / \Omega^{2}$ and $v_{T}=(T / M)^{1 / 2}$. When $\hat{\Phi}_{0}\left(k_{\perp} v_{\perp} / \Omega\right)$ is employed in the preceding integral the result is $\operatorname{Nexp}\left(-k_{\perp}^{2} v_{T}^{2} / \Omega^{2}\right) I_{0}\left(k_{\perp}^{2} v_{T}^{2} / \Omega^{2}\right)$ which reduces to 
the proper result for $k_{\perp}^{2} v_{T}^{2} / \Omega^{2}<1$ if the mnemonic $k_{\perp}^{2} \hat{\Phi} \rightarrow\left(k^{2}-\partial^{2} / \partial r^{2}\right) \hat{\Phi}$ is employed. 4

In appendix $A, a$ full electromagnetic derivation of the gyro-kinetic equations is presented which also retains an unperturbed electric field $\underline{E}_{0}=E_{0} \hat{\xi}$ and neoclassical, $F_{N}$, contributions to $F=F_{M}+F_{D}+F_{N}$. The vector potential $\underline{A}$ is written as $\underline{A}=A_{11} \underline{\hat{n}}+A_{r} \underline{\hat{r}}+\cdot A_{e} \hat{e}$ and no gauge is assumed. Employing $F_{N} / F_{M} \ll 1$, the neoclassjcal modifications are found to result in only small corrections to the Maxwellian contributions. As a result, eq. (5) is found to remain valid and eqs. (7) and (8) are replaced by

$$
\begin{aligned}
& \left\{-i \omega+\left[v_{11}+\frac{\mu B}{\Omega} \hat{\underline{n}} \cdot \nabla \times \underline{\hat{n}}\right] \underline{\hat{n}} \cdot \nabla_{s}+\underline{\hat{n}} \times\left[\frac{v_{n}}{\hat{\Omega}} \underline{\hat{n}} \cdot \nabla \underline{\hat{n}}+\frac{\mu}{\Omega} \nabla B\right] \cdot \nabla_{f}\right. \\
& \left.+\frac{Z e}{M} v_{11} E_{0} \frac{\partial}{\partial E}\right\} \hat{g}^{\prime}-\left\langle\exp (i L) C\left\{\hat{g}^{\prime} \exp (-i L)\right\}\right\rangle \\
& \left.=-\frac{i Z e F_{M}}{T}\left[\omega-\omega_{*}^{T}-\frac{i Z e}{T} E_{0} v_{11}\right]_{1}\right]_{0}\left(\frac{k v_{1}}{\Omega}\right)\left[\hat{\Phi}-\frac{v_{11}}{c} \hat{A}_{11}\right. \\
& \left.\left.+\frac{v_{d}}{\Omega} \sin \phi \frac{\partial \hat{\phi}}{\partial r}+\frac{v_{\perp}^{2}}{2 \Omega^{2}} \sin ^{2} \dot{\phi} \frac{\partial^{2} \hat{\phi}}{\partial r^{2}}\right]+J_{1}\left(\frac{k v_{\perp}}{\Omega}\right)\left[\frac{v_{\perp}}{2 k \Omega} \frac{\partial^{2} \hat{\phi}}{\partial r^{2}}+\frac{v_{\perp}}{k c} \frac{\partial \hat{A}_{e}}{\partial r}-\frac{i v_{j}}{c} \hat{A}_{r}\right]\right\} \\
& -\frac{Z^{2} e^{2} F_{M}}{T^{2}} v_{11} E_{0}\left[\frac{v_{11}}{c} \hat{A}_{11} J_{0}\left(\frac{k v_{\perp}}{\Omega}\right)-\left(\frac{v_{\perp}}{k \Omega} \frac{\partial \hat{A}_{e}}{\partial r}-\frac{i v_{\perp}}{c} \hat{A}_{r}\right) J_{1}\left(\frac{k v_{\perp}}{\Omega}\right)\right] \text {. }
\end{aligned}
$$

By employing eqs. (5) and (9) for drift waves in a sheared magnetic field the extension of the finite $\beta$ equations of references 3 and 4 to arbitrary $k v_{\alpha} / \Omega$ could be carried out by employing a Coulomb gauge for $\underline{A}$ in order to el iminate $\hat{A}_{e}$. A system of three coupled differential equations is obtained from Poisson's equation and the parallel and $\underline{\hat{r}}$ components of Ampere's law. The generality of the subsequent reduction of these finite $\beta$ equations to a system of two coupled second order differential equations in $\hat{\Phi}$ and $\hat{A}_{11}$ could be checked.

Equation (5), and (7) - (8) or (9), plus an appropriate collision operator 
are the basic equations that result from the gyro-kinetic ordering. In Appendix $B$ collision operators valid for arbitrary $k v_{1} / \Omega$ are obtained for like and unlike particle collisions.

For collisions of electrons with ions a Lorentz or pitch angle operator, eq. (B.6), may be employed. ${ }^{11-12}$ Changing to $\mu, E, \phi$ variables and gyroaveraging results in

$$
\left\langle\exp (i L) C_{e i}\left\{\hat{g}_{e}^{\prime} \exp (-i L)\right\}\right\rangle=\frac{4 \pi z_{i}^{2} e^{4} N_{j} \ln \Lambda}{m_{e}^{2} v^{3}} \frac{v_{11}}{B} \frac{\partial}{\partial \mu}\left[\mu v_{\|} \frac{\partial \hat{g}_{e}^{\prime}}{\partial \mu}\right] .
$$

For the collisions of ions with electrons $C_{i e} \approx 0$ may be employed because $c_{i e} \sim\left(m_{e} / M_{i}\right)^{1 / 2} c_{i, i}$ and the order $m_{e} / M_{i}$ momentum corrections from $c_{i e}$ may be neglected. In obtaining the gyro-averaged collision operators the distinction between the primed and unprimed variables may be ignored.

Eq. (11) does nor contain $\mathrm{kv}_{1} / \Omega$. corrections because only the perturbed electron distribution function enters. For ion-ion encounters, however, finite gyroradius corrections can be extremely important for $k v_{\alpha} / \Omega>1 .^{13}$ Transforming the first form of $(B 7)$ to $\mu, E, \phi$ and gyro-averaging, the like particle collision operator appropriate for arbitrary $\mathrm{kv}_{2} / \Omega$ is found to be

$$
\begin{aligned}
& \left\langle\exp (i L)\left\{C_{\ell}\left\{\hat{g}^{\prime} \exp (-i L)\right\}-P_{\ell^{\prime}} F_{M}\right\}=\right. \\
& \quad \frac{4 \pi Z^{4} e^{4} N \ell n \Lambda}{M^{2} v^{3}}\left\{[\operatorname{Erf}(x)-\psi(x)] \frac{v_{11}}{B} \frac{\partial}{\partial \mu}\left[\mu v_{11} \frac{\partial \hat{g}^{\prime}}{\partial \mu}\right]\right. \\
& +4 E^{2} \psi(x)\left[\frac{\partial^{2} g^{\prime}}{\partial E^{2}}+\frac{\mu}{E} \frac{\partial \hat{g}^{\prime}}{\partial \mu}\right]+2 v^{3}\left(\frac{M}{2 T}\right)^{3 / 2}\left[E r r^{\prime}(x)\right] \hat{g}^{\prime} \\
& : \\
& \left.-\frac{k^{2} E}{\Omega^{2}}\left[\operatorname{Erf}(x)-\psi(x)-\frac{\mu B}{2 E}(\operatorname{Erf}(x)-3 \psi(x))\right]\right\},
\end{aligned}
$$

where $x=v(M / 2 T)^{1 / 2}$; Erf, Erf', and $\psi$ are defined in appendix $B$; and from eqs. (B9), 


$$
\begin{aligned}
\left\langle\exp (i L) P_{\ell} F_{M}\right\rangle= & \frac{8 \pi Z^{4} e^{4} F_{M^{l n} \Lambda}}{T^{2}}\left\{v_{11} J_{0}^{2}\left(\frac{k v_{\perp}}{\Omega}\right) \int d^{3} v \frac{v_{11}}{v} \psi(x) \hat{g}^{\prime}\right. \\
& +v_{\perp} J_{1}^{2}\left(\frac{k v_{\perp}}{\Omega}\right) \int d^{3} v \frac{v_{\perp}}{v} \psi(x) \hat{g}^{\prime} \\
& \left.+\frac{1}{3}\left(E-\frac{3 T}{2 M}\right) J_{0}^{2}\left(\frac{k v_{\perp}}{\Omega}\right) \int d^{3} v \frac{\hat{g}^{\prime}}{v}\left[\operatorname{Erf}(x)-2 x \operatorname{Erf} f^{\prime}(x)\right]\right\} .
\end{aligned}
$$

For large $k^{2} T / M \Omega^{2}$, eqs. (12) and (13) simplify considerably so that for ionion collisions

$$
\begin{aligned}
\left\langle\exp (i L) C_{i j}\left\{\hat{g}_{j}^{\prime} \exp (-i L)\right\}\right\rangle & =-\frac{4 \pi Z_{i}^{4} e^{4} N_{j} \ln \Lambda}{M_{i}^{2} v^{3}} \frac{k^{2} E}{\Omega_{i}^{2}}\left[\operatorname{Erf}-\psi-\frac{\mu B}{2 E}(\operatorname{Erf}-3 \psi)\right] \\
& =-\frac{2 \pi Z_{i}^{4} e^{4} N_{i} \ln \Lambda}{M_{i}^{2} v} \frac{k^{2}}{\Omega_{i}^{2}} \operatorname{Erf}(x) .
\end{aligned}
$$

The last step follows by noting that to within factors of two Erf $-\psi-(\mu B / 2 E)($ Erf $-\psi) \approx$ Erf. For $k^{2} T / M \Omega^{2} \leq 1$ no similar simplification is apparent. For trapped particles a simplification results when the orderings $\partial / \partial E \sim 1 / E$ and $\partial / \partial \mu \sim 1 / \mu \sqrt{\varepsilon}$ are employed, where $\varepsilon$ is the inverse aspect ratio. Then eqs. (12) and (13) reduce to

$$
\begin{aligned}
& \left\langle\exp (i L) C_{\ell}\left\{g^{\prime} \exp (-i L)\right\}\right\rangle= \\
& \quad \frac{4 \pi Z^{4} e^{4} N \ell n \Lambda}{M^{2} v^{3}}\left\{[\operatorname{Erf}(x)-\psi(x)] \frac{v_{11}}{B} \frac{\partial}{\partial \mu}\left[\mu v_{11} \frac{\partial \hat{g}^{\prime}}{\partial \mu}\right]+4 \mu^{2} \psi(x) \frac{\partial^{2} \hat{g}^{\prime}}{\partial \mu^{2}}\right\} .
\end{aligned}
$$

This form is appropriate for the collisions of trapped particles with particles of the same species which are either trapped or circulating. 


\section{DISCUSSION}

By employing an ordering in which finite gyroradius corrections are retained to lowest order in the linearized Fokker-Planck equation, a description based on the notions of drift-kinetic theory is developed which is appropriate in sheared magnetic fields. The equation that results from this gyro-kinetic ordering is valid for an arbitrary ratio of poloidal wavelength to gyroradius. The result agrees with previous slab results ${ }^{2}$ and indicates that WKB or eikonal gyro-kinetic treatments ${ }^{8-9}$ must be employed with care for sheared magnetic fields. In addition, the full electromagnetic generalization of the gyro-kinetic equation is obtained with the unperturbed toroidal electric field modifications. Neoclassical corrections to the unperturbed distribution function have been retained also, but are shown to result in negligible corrections.

In order to obtain a like particle (ion-ion) collision operator valid for arbitrary value of poloidal wavenumber times gyroradius, the appropriate gyro-average of the Fokker-Planck collision operator is evaluated. The resulting expression is rather involved, but for large values of the gyroradius over poloidal wavelength is extremely simple. Other simplifications of the like particle collision operator may also be possible in certain limits; an additional example appropriate for trapped particles is noted. 


\section{APPENDIX A}

Retaining the vector potential $\underline{A}$, the neoclassical modification of the unperturbed distribution function $\mathrm{F}_{N}=\mathrm{F}_{N}(r, \theta, \mu, E)$, and the unperturbed electric field $E_{0}=E_{0} \hat{\zeta}$ replaces eq. (1) by

$$
\begin{aligned}
& \frac{\partial}{\partial t}\left[f-\frac{Z e}{M c} \underline{A} \cdot \nabla_{v} F\right]+\left[\underline{v} \cdot \nabla-\Omega \frac{\partial}{\partial \phi}\right]\left\{f-\frac{Z e \Phi}{M} \frac{\partial}{\partial E}\left(F_{M}+F_{N}\right)-\frac{Z e}{M B} \frac{\partial F_{N}}{\partial \mu}\left(\Phi-\frac{v_{11}}{c} A_{11}\right)\right\} \\
& -\frac{Z e}{M \Omega} \frac{\partial F_{M}}{\partial r} \underline{e} \cdot \nabla \Phi-Z e v_{+}\left[\Phi \cos \phi-\frac{1}{\Omega} \sin \phi \underline{v} \cdot \nabla \Phi\right] \frac{\partial}{\partial r}\left(\frac{F_{M}}{T}\right) \\
& +\frac{Z e v_{11}}{M B} \frac{\partial F_{N}}{\partial \mu} \underline{\hat{n}} \cdot \nabla\left(\Phi-\frac{v_{11}}{C} A_{11}\right)+\frac{Z e}{M} \Phi \underline{v} \cdot \nabla\left(\frac{\partial F_{N}}{\partial E}+\frac{1}{B} \frac{\partial F_{N}}{\partial \mu}\right)-\frac{Z e v_{11}}{M C} A_{11}-\nabla \cdot \nabla \frac{1}{B}\left(\frac{\partial F_{N}}{\partial \mu}\right) \\
& +\frac{Z e}{M c}(\nabla \underline{A} \cdot \underline{v}-\underline{v} \cdot \nabla \underline{A}) \cdot \nabla_{v} F_{D}-\frac{Z e}{M c}\left[v_{11} \underline{\hat{n}} \cdot \nabla \underline{A} \cdot \underline{v}_{1}+\underline{v}_{+} \cdot \nabla\left(\underline{\hat{n}} v_{11}\right) \cdot \underline{A}\right] \frac{1}{B} \frac{\partial F_{N}}{\partial \mu} \\
& +\frac{Z e}{M} \underline{\hat{\zeta}} \cdot \underline{v E}_{0} \frac{\partial f}{\partial E}+\left[\dot{\mu}+\frac{Z e}{M B} \underline{\underline{\zeta}} \cdot \underline{v}_{\perp} E_{0}\right] \frac{\partial f}{\partial \mu}+\left[\dot{\phi}+\frac{Z e}{M v_{\perp}} \underline{\zeta}^{\prime} \underline{\phi}_{0}\right] \frac{\partial f}{\partial \phi}=c\{f\} .
\end{aligned}
$$

In obtaining eq. (AI), $\nabla_{V} F_{D}$ from the text and

$$
\begin{aligned}
\underline{v} \times(\nabla \times \underline{A}) \cdot \underline{\hat{n}} \frac{v_{11}}{B} \frac{\partial F_{N}}{\partial \mu}= & v_{11} A_{11} \underline{v} \cdot \nabla\left(\frac{1}{B} \frac{\partial F_{N}}{\partial \mu}\right)-\underline{v} \cdot \nabla\left(\frac{v_{11} A_{11}}{B} \frac{\partial F_{N}}{\partial \mu}\right) \\
& +\left[v_{11} \underline{\hat{n}} \cdot \nabla\left(v_{11} A_{11}\right)+v_{11} \underline{\hat{n}} \cdot \nabla \underline{A} \cdot \underline{v}_{\perp}+\underline{v}_{\perp} \cdot \nabla\left(\underline{\hat{n}} v_{11}\right) \cdot \underline{A}\right] \frac{1}{B} \frac{\partial F_{N}}{\partial \mu}
\end{aligned}
$$

have been employed.

In addition to the small terms retained in eq. (4) it is now convenient to retain certain neoclassical terms to lowest order, with the result that

$$
\begin{gathered}
\left\{\left[\tilde{v}, \underline{r} \cos \phi+v_{\perp} \hat{e} \sin \phi\right] \cdot \nabla_{f}-\Omega \frac{\partial}{\partial \phi}+i \tilde{v}_{\perp} \cos \phi \frac{\partial L}{\partial r}\right\}\left\{\hat{f}-\frac{Z e \hat{\Phi}}{M} \frac{\partial}{\partial E}\left(F_{M}+F_{N}\right)\right. \\
\left.-\frac{Z e}{M B} \frac{\partial F_{N}}{\partial \mu}\left(\hat{\Phi}-\frac{v_{11}}{c} \hat{A}_{11}\right)\right\}=-\frac{Z e v_{11}}{M B} \frac{\partial F_{N}}{\partial \mu} \hat{n} \cdot \nabla_{f}\left(\hat{\Phi}-\frac{v_{11}}{c} \hat{A}\right) \equiv 0 .
\end{gathered}
$$


Changing eq. (A2) to the primed variables results in

$$
\hat{f}-\frac{Z e \hat{\Phi}}{M} \frac{\partial}{\partial E}\left(F_{M}+F_{N}\right)-\frac{Z e}{M B} \frac{\partial F_{N}}{\partial \mu}\left(\hat{\Phi}-\frac{V_{11}}{c} \hat{A}_{11}\right)=\hat{g}^{\prime} \exp (-i L)
$$

where again $\hat{g}^{\prime}$ is independent of $\phi^{\prime}$.

The next order equation is obtained from eq. (Al) in the same manner as the electrostatic case. Upon carrying out the primed gyrophase average the resulting equation has the left hand side shown in eq. (9). Retaining only terms that result in non-negligible contributions the right hand side gives

$$
\begin{aligned}
& \frac{i Z e}{M}<\left\{\left[\omega \frac{\partial}{\partial E}\left(F_{M}+F_{N}\right)-\omega_{\star}^{T} \frac{\partial F_{M}}{\partial E}\right] \hat{\Phi}+\frac{i Z e}{M} \underline{\zeta} \cdot \underline{v E}_{0} \frac{\partial}{\partial E}\left[\hat{\Phi} \frac{\partial}{\partial E}\left(F_{M}+F_{N}\right)+\left(\hat{\Phi}-\frac{v_{11}}{c} \hat{A}_{11}\right) \frac{1}{B} \frac{\partial F_{N}}{\partial \mu}\right]\right. \\
& +\frac{1}{B} \frac{\partial F_{N}}{\partial \mu}\left[\left(\omega+i v_{11} \underline{\hat{n}} \cdot \nabla_{s}\right)\left(\hat{\Phi}-\frac{v_{11}}{c} \hat{A}_{11}\right)\right]+i \hat{\Phi} \underline{v} \cdot \nabla\left(\frac{\partial F_{N}}{\partial E}+\frac{1}{B} \frac{\partial F_{N}}{\partial \mu}\right)-\frac{i v_{11}}{c} \hat{A}_{11} \underline{v} \cdot \nabla\left(\frac{1}{B} \frac{\partial F_{N}}{\partial \mu}\right) \\
& \left.\left.-\frac{\omega}{c} \underline{\hat{A}} \cdot \nabla_{v} F+\frac{i}{c}\left[\nabla_{f} \underline{\hat{A}} \cdot \underline{v}-\underline{v} \cdot \nabla_{f} \underline{\hat{A}}\right] \cdot \nabla_{v} F_{D}\right\} \exp (i L)\right\rangle= \\
& \frac{i Z e}{M}\left\{\left[\omega \frac{\partial}{\partial E}\left(F_{M}+F_{N}\right)-\omega_{*}^{T} \frac{\partial F_{M}}{\partial E}+\frac{i Z e v_{n} E_{0}}{M B} \frac{\partial}{\partial E}\left(\frac{\partial F_{N}}{\partial \mu}\right)\right]\left[\langle\hat{\Phi} \exp (i L)\rangle-\frac{v_{11}}{c} \hat{A}_{11} J_{0}\right]\right. \\
& +\frac{i Z e}{M} v_{n} E_{0}\left[\frac{\partial^{2}}{\partial E^{2}}\left(F_{M}+F_{N}\right)\right]\langle\hat{\Phi} \exp (i L)\rangle \\
& +\left[\omega\left(\frac{\partial F_{M}}{\partial E}+\frac{\partial F_{N}}{\partial E}+\frac{1}{B} \frac{\partial F_{N}}{\partial \mu}\right]-\omega_{\star}^{T} \frac{\partial F_{N}}{\partial E}\right]\left[\frac{v_{1}}{k c} \frac{\partial \hat{A}_{e}}{\partial r}-\frac{i v_{\perp}}{c} \hat{A}_{r}\right] J_{1} \\
& +\frac{1}{B} \frac{\partial F_{N}}{\partial \mu}\left[\omega\left(\langle\hat{\Phi} \exp (i L)\rangle-\frac{V_{11}}{c} \hat{A}_{11} J_{0}\right)+J_{0} i v_{11} \hat{\hat{n}} \cdot \nabla_{S}\left(\hat{\Phi}-\frac{v_{11}}{C} \hat{A}_{11}\right)\right] \\
& \left.-v_{\perp} J_{1}\left[\hat{\Phi} \frac{\partial}{\partial r}\left(\frac{\partial F_{N}}{\partial E}\right)+\left(\hat{\Phi}-\frac{v_{11}}{c} \hat{A}_{11}\right) \frac{\partial}{\partial r}\left(\frac{1}{B} \frac{\partial F_{N}}{\partial \mu}\right)\right]\right\} \text {. }
\end{aligned}
$$

From eqs. (A3) and (A4) the importance of the neoclassical terms can be 
16

estimated. Employing $F_{N} / F_{M} \sim(q / \varepsilon)\left(v_{1} / \Omega\right)\left|N^{-1} \partial N / \partial r\right| \ll 1$ and noting that the $\left(\omega+\underline{i n} \cdot \nabla_{s}\right)\left(\hat{\Phi}-v_{11} c^{-1} \hat{A}_{11}\right)$ terms of (A4) can be combined with the terms on the left hand side, the neoclassical terms are seen to result in small corrections to the Maxwellian contributions. As a result, eq. (A3) reduces to eq. (5) and eq. (A4) becomes

$$
\begin{aligned}
\frac{i Z e}{M}\left\{\frac{\partial F_{M}}{\partial E}\left(\omega-\omega_{*}^{\top}\right)\right. & {\left[\langle\hat{\Phi} \exp (i L)\rangle-\frac{v_{11}}{c} A_{11} J_{0}+\left(\frac{v_{1}}{k c} \frac{\partial \hat{A}_{e}}{\partial r}-\frac{i v_{\perp}}{c} \hat{A}_{r}\right) J_{1}\right.} \\
+ & \left.\frac{i Z e E_{0}}{M} v_{11} \frac{\partial^{2} F_{M}}{\partial E^{2}}\langle\hat{\Phi} \exp (i L)\rangle\right\} .
\end{aligned}
$$

Expression (9) then follows by substituting eq. (8) into (A5). 
The pitch angle scattering collision operator of Rosenbluth, Hazeltine, and Hinton!" and/or Rosenbluth, Ross and Kostomarov ${ }^{12}$ can no longer be safely employed because operations on the $\exp (-i L)$ in $c\left\{\hat{g}^{\prime} \exp (-i L)\right\}$ can result in terms which must be retained for $k v_{1} / \Omega \geq 1$. As a result, an appropriate collision operator will be derived by starting with the full Fokker-Planck operator ${ }^{14}$

$$
\left.\frac{d \mathscr{F}}{d t}\right|_{c 011}=\sum^{\prime} \Gamma^{\prime} \nabla_{v} \cdot\left\{\nabla_{v} \cdot\left[\frac{1}{2} F \nabla_{v} \nabla_{v} G\right]-F \nabla_{v} H\right\}
$$

with

$$
\begin{aligned}
& H=H(\underline{v})=\frac{M+M^{\prime}}{M^{\prime}} \int d^{3} v^{\prime} \frac{F^{\prime}}{\underline{\underline{v}-\underline{v}^{\prime} T}} \\
& \nabla_{v^{2}}^{2}=-4 \pi \frac{M+M^{\prime}}{M^{\prime}} \mathcal{F}^{\prime} \\
& G=G(\underline{v})=\int d^{3} v^{\prime}\left|\underline{v}-\underline{v}^{\prime}\right| \mathcal{F}^{\prime} \\
& \nabla_{v^{2}}^{2}=\frac{2 M^{\prime}}{M+M^{\prime}} H, \\
& \Gamma^{\prime}=\left(4 \pi z^{2} z^{\prime} e^{4} / M^{2}\right) \ln \Lambda,
\end{aligned}
$$

where $F$ is the total (unperturbed plus perturbed) distribution function and primes denote the species integrated over all velocities. The sum is over all primed species and must include like particle collisions; $\ln \Lambda$ is the Coulomb logarithm.

Linearizing by writing $\mathcal{F}=F_{M B}+h$, where

$$
F_{M B}=F_{M} \exp (-Z e \Phi / T) \approx F_{M}\left[1-\frac{Z e}{T} \Phi\right]
$$




$$
h=\hat{g}^{\prime} \exp (-i L)
$$

results in

$$
\begin{aligned}
c[h] & =\sum^{\prime} \exp \left(-\frac{Z^{\prime} e \Phi}{T^{\prime}}\right)\left[\Gamma^{\prime} K^{\prime}+P^{\prime}(\underline{v}) F_{M}\right] \\
& \approx \Sigma^{\prime}\left[\Gamma^{\prime} K^{\prime}+P^{\prime}(\underline{v}) F_{M}\right]
\end{aligned}
$$

with

$$
\begin{aligned}
& K^{\prime}=\nabla_{v} \cdot\left\{\nabla_{v} \cdot\left[\frac{1}{2} h \nabla_{v} \nabla_{v} G\right]-h \nabla_{v} H\right\} \\
& H=\frac{N^{\prime}}{v} \frac{\left(M+M^{\prime}\right)}{M^{\prime}} \operatorname{Erf}\left[v\left(\frac{M^{\prime}}{2 T^{\prime}}\right)^{1 / 2}\right] \\
& G=N^{\prime}\left\{\left(v+\frac{T^{\prime}}{M^{\prime} v}\right) \operatorname{Erf}\left[v\left(\frac{M^{\prime}}{2 T^{\top}}\right)^{1 / 2}\right]+\left(\frac{T^{\prime}}{2 M^{\prime}}\right]^{1 / 2} \operatorname{Erf}{ }^{\prime}\left[v\left(\frac{M^{\prime}}{2 T^{\prime}}\right)^{1 / 2}\right]\right\} \\
& \operatorname{Erf}(x)=\frac{2}{\pi^{1 / 2}} \int_{0}^{x} d t \exp \left(-t^{2}\right) \\
& \operatorname{Erf}(x)=\frac{2}{\pi^{1 / 2}} \frac{d}{d x} \int_{0}^{x} d t \exp \left(-t^{2}\right)=\frac{2}{\pi^{1 / 2}} \exp \left(-x^{2}\right),
\end{aligned}
$$

where $H$ and $G$ have been evaluated using $F_{M}$ and $v=|\underline{v}|$. The functional of $h^{\prime}, P^{\prime} F_{M}$, is in general proportional to $\nabla_{v} \cdot\left\{\nabla_{v} \cdot\left[\frac{1}{2} F_{M} \nabla_{v} \nabla_{v} G\right]-F_{M} \nabla_{v} H\right.$ with $G$ and $H$ evaluated using $h^{\prime}$; however, rather than use such a complicated form is it convenient to employ a simpler $\mathrm{P}^{\prime}$ determined by the conservation of momentum and energy constraints. The model of eq. (B2) may also ${ }^{11}$ be thought of as somewhat similar to a Bhatnagar-Gross-Krook model in that the momentum and energy removed by the $K^{\prime}$ term is replenished in a Maxwellian distribution by the $P^{\prime} F_{M}$ term.

Employing the properties of $G$ and $H$ listed in eqs. (BI), $K^{\prime}$ may be 
rewritten as

$$
K^{\prime}=\frac{1}{2} \nabla_{v} \nabla_{v} G: \nabla_{v} \nabla_{v} h+\frac{M^{\prime}-M}{M^{\prime}+M} \nabla_{v} H^{\prime} \cdot \nabla_{v} h+4 \pi \frac{M}{M^{\prime}} h F_{M}^{\prime}
$$

For $F^{\prime}=F_{M^{\prime}}^{\prime}, G$ and $H$ are functions of $v$ only, as indicated in eqs. (B3). As a result ' $K$ ' may be rewritten once again as

$$
\begin{aligned}
K^{\prime}=\left\{\left[\frac{\underline{\underline{I}} v^{2}-3 \underline{v} \underline{v}}{2 v^{3}}\right] \frac{\partial G}{\partial v}\right. & \left.+\frac{M^{\prime} H}{M+M^{\prime}} \frac{\underline{v}}{v^{2}}\right\}: \nabla \nabla v^{2} v^{h} \\
& +\frac{M^{\prime}-M}{M^{\prime}+M} \frac{1}{v} \frac{\partial H}{\partial v} \underline{v} \cdot \nabla v h+4 \pi \frac{M}{M^{\prime}} F_{M^{\prime}} h,
\end{aligned}
$$

where $I$ is the unit dyadic.

To simplify $K^{\prime}$ further, spherical velocity variables $v=|\underline{v}|, \alpha$, and $\phi$ defined with respect to $\underline{B}_{0}=\underline{B} \underline{n}$ are employed: The angles $\alpha$ and $\phi$ are the pitch angle $\tan ^{-1}\left(v_{\perp} / v_{11}\right)$ and the gyrophase, respectively; the unit vectors form a right handed orthogonal system in which $\underline{\hat{v}} \times \underline{\hat{\alpha}}=\hat{\Phi}$. Because of the form of eq. (B4), only terms in $\underline{\hat{v}} \underline{\hat{v}}, \underline{\hat{\alpha}} \underline{\underline{\alpha}}$, and $\hat{\Phi} \hat{\Phi}$ need be retained when forming $\nabla_{v} \nabla_{v} h$; all other terms give zero when double dotted into $\underline{v} \underline{v}$ and $\underline{\underline{I}}=\underline{\hat{v}} \underline{\hat{v}}+\underline{\hat{\alpha}} \hat{\underline{\alpha}}+\hat{\phi} \hat{\phi}$. Carrying out the transformation to spherical velocity variables results in

$$
\begin{aligned}
K^{\prime}= & \frac{1}{2 v^{3}} \frac{\partial G}{\partial v}\left\{\frac{1}{\sin \alpha} \frac{\partial}{\partial \alpha}\left(\sin \alpha \frac{\partial h}{\partial \alpha}\right)+\frac{1}{\sin ^{2} \alpha} \frac{\partial^{2} h}{\partial \phi^{2}}\right\}+4 \pi \frac{M}{M^{\prime}} F_{M^{\prime}}^{h} \\
& +\left[\frac{M^{\prime} H}{M+M^{\prime}}-\frac{1}{v} \frac{\partial G}{\partial v}\right] v \frac{\partial}{\partial v}\left[\frac{1}{v} \frac{\partial h}{\partial v}\right]+\left[\frac{M^{\prime} H}{M+M^{\prime}}+\frac{M^{\prime}-M_{i}}{M^{\prime}+M} v \frac{\partial H}{\partial v}\right] \frac{1}{v} \frac{\partial h}{\partial v} .
\end{aligned}
$$

At this point is it convenient to consider unlike and like particle collisions separately. In particular, for the collisions of electrons with ions a Lorentz collision operator will be employed. As a result, collisions of electrons with ions will automatically conserve number and energy, and the 
electrons may transfer as much momentum to the ions as they wish. Consequently, for these electron-ion collisions the $P^{\prime}$ of eq. (B2) is zero to the order of interest. In addition, for the collisions of ions with electrons $C_{j e}=0$ may be employed because $C_{i e} \sim\left(m_{e} / M_{j}\right)^{1 / 2} C_{i j}$.

For the collisions of electrons $\left(M \rightarrow m_{e}, Z \rightarrow-1, T \rightarrow T_{e}, h \rightarrow h_{e}\right)$ with ions $\left(N^{\prime} \rightarrow N_{j}, M^{\prime} \rightarrow M_{j}, Z^{\prime}+Z_{j}, T^{\prime}+T_{j}, F_{M} \rightarrow F_{M i}\right), H \approx N^{\prime} / v, \partial H / \partial v \approx-N^{\prime} / v^{2}$ and $\partial G / \partial V \approx N^{\prime}$ may be employed to obtain the Lorentz gas collision operator $\left(P^{\prime} \rightarrow 0\right)$

$$
c_{e i} \approx \frac{2 \pi Z_{i}^{2} e^{4} N_{j} \ln \Lambda}{m_{e}^{2} v^{3}}\left[\frac{1}{\sin \alpha} \frac{\partial}{\partial \alpha}\left(\sin \alpha \frac{\partial h_{e}}{\partial \alpha}\right)+\frac{1}{\sin ^{2} \alpha} \frac{\partial^{2} h_{e}}{\partial \phi^{2}}\right],
$$

where mass ratio corrections are neglected. From the form of eq. $(B \overline{6})$, conservation of number and energy are apparent.

For like particle collisions, employing the expressions for $G$ and $H$ rruill eys. (B3) âliows eq. (B5) to be written as

$$
\begin{aligned}
C_{\ell}-P_{\ell} F_{M}= & \frac{N \Gamma}{2 v^{3}}[\operatorname{Erf}(x)-\psi(x)]\left[\frac{1}{\sin \alpha} \frac{\partial}{\partial \alpha}\left(\sin \alpha \frac{\partial h}{\partial \alpha}\right)+\frac{1}{\sin ^{2} \alpha} \frac{\partial^{2} h}{\partial \phi^{2}}\right] . \\
& +N \Gamma\left[\psi(x) \frac{\partial}{\partial v}\left(\frac{1}{v} \frac{\partial h}{\partial v}\right)+\operatorname{Erf}(x) \frac{1}{v^{2}} \frac{\partial h}{\partial v}+2\left(\frac{M}{2 T}\right)^{3 / 2} h \operatorname{Erf}^{\prime}(x)\right] \\
= & \frac{N \Gamma}{2 v^{3}}[\operatorname{Erf}(x)-\psi(x)]\left[\frac{1}{\sin \alpha} \frac{\partial}{\partial \alpha}\left(\sin \alpha \frac{\partial h}{\partial \alpha}\right)+\frac{1}{\left.\sin ^{2} \alpha \frac{\partial^{2} h}{\partial \phi^{2}}\right]}\right. \\
& +\frac{N \Gamma}{v^{2}} \frac{\partial}{\partial v}\left\{v \psi(x) \frac{\partial h}{\partial v}+[\operatorname{Erf}(x)-2 \psi(x)] h-v \frac{\partial \psi(x)}{\partial v} h\right\},
\end{aligned}
$$

where $x=v(M / 2 T)^{1 / 2}$ and $\psi(x) \equiv\left(1 / 2 x^{2}\right)\left[\operatorname{Erf}(x)-x \operatorname{Erf}^{\prime}(x)\right]$. The second form of equation (B7) follows by noting that 


$$
\frac{\partial}{\partial v}\left[v \frac{\partial \psi(x)}{\partial v}\right]-\frac{\partial}{\partial v}[\operatorname{Erf}(x)-2 \psi(x)]+2 \frac{x^{3}}{v} \operatorname{Erf}^{\prime}(x) \equiv 0
$$

This latter form of eq. (B7) can then be rewritten in a form more convenient for determining conservation properties, namely,

$$
\begin{aligned}
C_{\ell}-P_{\ell} F_{M}= & \nabla_{v} \cdot\left\{\frac { 2 \pi Z ^ { 4 } e ^ { 4 } N \ell n \Lambda } { M ^ { 2 } } \left[\nabla_{v}\left([\operatorname{Erf}(x)-\psi(x)] \frac{h}{v}\right)\right.\right. \\
\therefore \quad & \left.\left.+\frac{\hat{v}}{v^{2}}\left[\frac{\partial}{\partial v}\{v[3 \psi(x)-\operatorname{Erf}(x)] h\}+8 x^{2} \psi(x) h\right]\right]\right\}
\end{aligned}
$$

In this form conservation of number is automatic, and $P_{\ell}$ is determined from conservation of momentum and energy. Taking $P_{\ell}=\underline{v} \cdot \underline{p}_{l}+\lambda_{l}\left[\left(M v^{2} / 2 T\right)-\frac{3}{2}\right]$, $p_{\ell}$ and $\lambda_{\ell}$ are determined from conservation of momentum and energy, respectively,

$$
\begin{gathered}
\underline{R}_{\ell}=\frac{8 \pi Z^{4} e^{4} \ell n \Lambda}{T^{2}} \int d^{3} v \underline{\hat{v}} \psi(x) h \\
\lambda_{\ell}=\frac{8 \pi Z^{4} e^{4} \ell n \Lambda}{3 M T} \int d^{3} v \frac{h}{v}\left[\operatorname{Erf}(x)-2 x E r f^{\prime}(x)\right] .
\end{gathered}
$$




\section{REFERENCES}

1. L. D. Pearlstein and H. L. Berk, Phys. Rev. Lett. 23, 220 (1969).

2. M. N. Rosenbluth and P. J. Catto, Nuclear Fusion 15, 573 (1975).

3. P. J. Catto, A. M. El-Nadi, C. S. Liu, and M. N. Rosenbluth, Nuclear Fusion 14, 405 (1974).

4. W. M. Tang, C. S. Liu, M. N. Rosenbluth, P. J. Catto, and J. D. Callen, to be published in Nuclear Fusion, and Princeton Plasma Physics Laboratory Report MATT-1153, 1975.

5. K. T. Tsang and J. D. Callen, Proceedings of Annual Meeting on Theoretical Aspects of Controlled Thermonuclear Research, Arlington, VA, April 1975. and F. L. Hinton and David W. Ross, Fusion Research Center Report FRCR No. 91, Univ. of Texas at Austin, Ju1y, 1975.

6. P. J. Catto, K. T. Tsang, J. D. Callen, and W. M. Tang, submitted to Phys. Fluids, and ORNL-TM-5158 (Nov. 1975).

C. S. Liu, M. N. Rosenbluth, and W. M. Tang, Princeton Plasma Physics Laboratory Report MATT-1125.

W. M. Tang, P. H. Rutherford, H. P. Furth, and J. C. Adam, Phys. Rev. Lett. $35,660(1975)$.

7. P. H. Rutherford and E. A. Frieman, Phys. Fluids 11, 569 (1968).

8. A detailed finite beta derivation employing the technique of reference 8 has been carried out by B. Newberger and P. H. Rutherford (private communication).

9. E. Jamin, Ph.D. Thesis, Princeton University (1971).

10. See, for example, Appendix $A$ of reference 2, between eqs. (A7) and (A8).

11. M. N. Rosenbluth, R. D. Hazeltine, and F. L. Hinton, Phys. Fluids 15, $116(1972)$. 
12. M. N. Rosenbluth, D. W. Ross, and D. P. Kostomarov, Nuclear Fusion 12, 3 (1973).

13. L. P. Pitaevskii, Sov. Phys. - JETP 17, 658 (1963) [Zh. Eksp. Theo. Fiz. 44, 469 (1963)] and A. A. Rukhadze and V. P. Silin, Sov. Phys. - Uspekhi 11, 659 (1969) [Usp. Fiz. Nauk 96, 87 (1968)].

14. M. N. Rosenbluth, W. M. MacDonald, and D. L. Judd, Phys. Rev. 107, 1 (1957). 
THIS PAGE

\section{WAS INTENTIONALLY \\ LEFT BLANK}




\section{INTERNAL DISTRIBUTION}

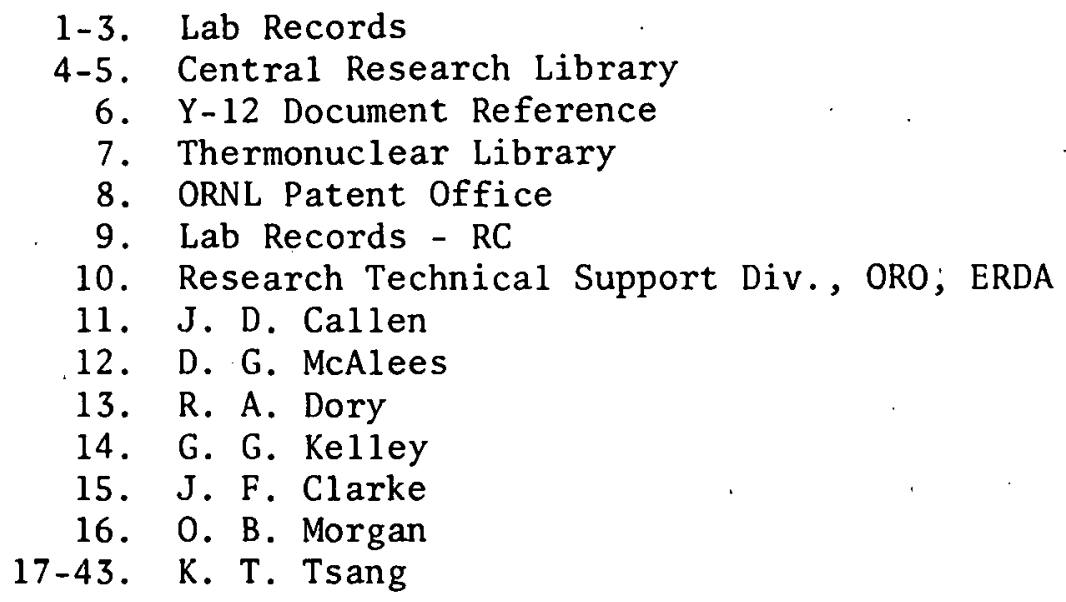

EXTERNAL DISTRIBUTION

44. Plasma Physics Library, Plasma Physics Laboratory, Princeton University, Forrestal Campus, P.0. Box 451, Princeton, NJ 08540

45. Controlled Thermonuclear Research Library, Lawrence Livermore Laboratory, P.0. Box 808, Livermore, CA 94550

46. Q Division Library, c/o F. L. Ribe, Los Alamos Scientific Laboratory, P.0. Box 1663, Los Alamos, NM 87544

47. Controlled Thermonuclear Research Library, c/o Weston M. Stacey, $\mathrm{Jr}$., Argonne National Laboratory, $9700 \mathrm{~S}$. Cass Ave., Argonne, IL 60439

48. CTR Computer Center, c/o Dr. John Killeen, Lawrence Livermore Laboratory, P.0. Box 808, Livermore, CA 94550

49. Librarian, Culham Laboratory, U.K. Atomic Energy Authority, Abingdon, Uxon, UX14, 3DB, United Kingdom

50. Ruth Lengye, Bibliothek, Max Planck Institut fur Plasmaphysik, 8046 Garching be Munchen, Federal Republic of Germany

51. Library, Centre de Recherches en Physique des Plasma, 21 Avenue des Bains, 1007, Lausanne, Switzerland

52. A. M. Dupas, Documentation S.I.G.N., Department de La Physique du Plasma, Et de La Fusion Controlee, Association EURATOM-CEA, Sur La Fusion, Centre D-Etudes Nucleaires, BP 85 Centre Du TRI 38041 Grenoble Cedex (FRANCE)

53. Bibliotheque, Service du Confinement des Plasmas, C.E.A., B.P. No. 6,92 , Fontenay-Aux-Roses (Seine) France 
54. Library, International Centre for Theoretical Physics, Trieste, Italy

55. Library, Laboratorio Gas Ionizati, Frascatti, Italy

56. V. E. Ivanov, Physical-Technical Institute of the Ukranian Academy of Sciences Sukhumi, U.S.S.R.

57. M. S. Rabinovich, Lebedev Institute of Physics, Academy of Sciences of the U.S.S.R., Leninsky Prospect 53, Moscow, U.S.S.R.

58. Thermonuclear Laboratory, Kurchatov Institute of Atomic Energy, 46 U1itsa Kurchatova, P. 0. Box 3402, Moscow, U.S.S.R.

59. Library; Institute for Plasma Physics, Nagoya University, Nagoya, Japan 464

60. Library, FOM-Institut voor Plasma-Fysica, Rijnhuizen, Jutphaas, Netherlands

61. Plasma Physics Group, Department of Engineering Physics, Australian National University, P.O. Box 4, Canberra A.C.T. 2600 Australia

62. Thermonuclear Library, Japan Atomic Energy Research Institute, Tokai, Naka, Ibaraki, Japan

63. CTR Reading Room, c/o Prof. Dieter J. Sigmar, Room 37-391,M.I.T., Cambridye, MA 02139 .

64. CTR Reading Room, c/o Prof. D. W. Kerst, Dept. of Physics, Sterling Ha11, Univ. of Wisconsin, Madison, WI 53706

65. CTR Reading Room, c/o Prof. I. B. Bernstein, Yale University, New Haven, Conn. 06510

66. Center for Plasma Physics and Thermonuclear Research, Univ. of . Texas, Physics Building 330, Austin, TX 78712

67. CTR Reading Room, c/o Prof. B. D. Fried, Physics Dept., Univ. of California, Los Angeles, CA 90024

68. CTR Reading Room, c/o Prof. David C. Montgomery, Physics \& Astronomy Dept., Univ. of Iowa, Iowa City, Iowa 52240

69. Magneto-Fluid-Dynamics Library, Courant Inst. of Math. Sci., New York Univ., 251 Mercer St., New York, NY 10012

70. CTR Reading Room, c/o Prof. Allan N. Kaufman, Physics Dept., Univ. of California, Berkeley, CA 94720

71. CTR Reading Room, c/o Prof. W. B. Thompson, Univ. of California, San Diego, Physics Dept., La Jolla, CA 92037 
72. CTR Reading Room, c/o Prof. Alvin W. Trivelpiece, Dept. of

Physics \& Astronomy, Univ. of Maryland, College Park,. MD 20742

73. CTR Reading Room, c/o Prof. T. Kammash, 103 Research Admin. B1dg., N. Campus, Univ. of Michigan, Ann Arbor, MI 48105

74. CTR Reading Room, c/o Dr. Ravi N. Sudan, Phillips Hall, Cornell Univ., Ithaca, NY 18450

75. Prof. Marshal1 N. Rosenbluth, Institute for Advanced Study, Princeton, NJ 08540

76. CTR Reading Room, c/o Prof. R. Gross, Plasma Research Lab., Columbia Univ., New York, NY 10027

77. CTR Reading Room, c/o Prof. Roy Gould, California Inst. of Tech., Pasadena, CA 91103

78. Dr. Nicholas A. Kral1, Science Applications, Inc., P.0. Box. 2354, 1200 Prospect St., La Jolla, CA 92037

79. CTR Reading Room, c/o Dr. Jay P. Boris, Plasma Physics, Naval Research Laboratory, Washington, DC 20390

80. CTR Library, General Atomic Co., P.0. Box 81608, San Diego, CA 92138

81. CTR Library, c/o Dr. Alan F. Haught, United Technologies Research Labs, East Hartford, Conn. 06108

82. Dr. Robert E. Price, Division of Controlled Thermonuclear Research, U. S. Energy Research and Development Administration, Washington, DC 20545

83. Dr. Bennett Miller, Division of Controlled Thermonuclear Research, U. S. Energy Research and Development Administration, Washington, DC 20545

84. Dr. Arthur Sleeper, Division of Controlled Thermonuclear Research, U. S. Energy Research and Development Administration, Washington, DC 20545

85. Dr. Walter Sadowski, Division of Controlled Thermonuclear Research, U. S. Energy Research and Development Administration, Washington, DC 20545

86. Dr. D. H. Priester, Divisiun of Controlled Thermonuclear Research U. S. Energy Research and Development Administration, Washington, DC 20545

87. Dr. Paul H. Rutherford, Princeton Plasma Physics Lab., Princeton Univ., Princeton, $\mathrm{NJ} 08540$ 
88. Dr. L. D. Pearlstein, L-388, Lawrence Livermore Laboratory, P.0. Box 808, Livermore, CA 94550

89. Dr. J. P. Friedberg, Los Alamos Scientific Laboratory, Los Alamos NM 87544

90. Dr. David W. Ross, Center for Plasma Physics \& Thermonuclear Research, Dept. of Physics, Univ. of Texas, Austin, TX 78712

91. Dr. Gareth E. Guest, General Atomic Co., P.0. Box 81608, San Diego, CA 92138

92. Dr. Claude Mercier, Service du Theorie des Plasmas, Centre d'Etudes Nucleaires, Fontenay-aux-Roses (Seine) France

93. Dr. J. B. Taylor, Culham Laboratory, UKAEA Abingdon, Oxon, 0x14 3DB, United Kingdom

94. Dr. D. Pfirsch, Institute for Plasma Physics, 8046 Garching bei Munchen, Federal Republic of Germany

95. Dr. V. D. Shafranov, I. V. Kurchatov Inst. of Atomic Energy, 46 Ulitsa Kurchatova, P.0. Box 3402, Moscow, U.S.S.R.

96. Dr. Harold Grad, Courant Inst. of Math. Sci., New York Univ., 251 Mercer St., New York, NY 10012

97. Dr. J. G. Cordey, Culham Laboratory, UKAEA, Abingdon, Oxon, 0x14, 3DB, United Kingdom

98. Dr. David Baldwin, L-388, Lawrence Livermore Lab., P.0. Box 808, Livermore, CA 94550

99. Prof. Bruno Coppi, Dept. of Physics, M. I.T., Cambridge, Mass. 02139

100. Dr. Harold P. Furth, Princeton Plasma Physics Lab., Princeton Univ., P.0. Box 451, Princeton, NJ 08540

101-127. Tech. Info. Center ERDA 